\section{A PRELIMINARY SURVEY: SYMPTOMS AND SYMPTOM CONTROL OF DYING INFANTS IN THE NICU IN TAIWAN}

\author{
N.-H. Peng ${ }^{1}$, C.-H. Chen², S.-L. Liu ${ }^{3}$, \\ H.-Y. Ho-Yu Lee ${ }^{4}$
}

${ }^{1}$ Private, ${ }^{2}$ Diversion of Neonatology in Taichung Veterans General Hospital in Taiwan, ${ }^{3}$ Nursing College in Central Taiwan University of Science and Technology in Taiwan, ${ }^{4}$ Nursing Department in Chang Bing Show Chwan Memorial Hospital,

Taichung, Taiwan R.O.C.

Aim: To describe the symptoms characteristic of dying neonates and document the neonatal clinical interventions were offered to dying infants and their families.

Background: Despite the great need for quality endof-life care for neonates and their families, there is very little information about either symptomatology of dying neonates as a group, or the medical treatments and nursing interventions provided for dying infants and their families.

Design: A retrospective chart review was used in this research. All charts of neonatal inpatients who died in the neonatal intensive care unit (NICU) between 2002 and 2008.

Results: Sixty-one charts were evaluated in this research. The major underlying disease was the complications of prematurity (33\%). Major physiological distress at the last week of life included respiratory distress $(67.2 \%)$, bradycardia (36.1\%), cyanosis $(54.1 \%)$, gastro-intestinal bleeding $(37.7 \%)$ and edema $(37.7 \%)$. All infants were intubated and received artificial ventilation in the last week of life. Physicians prescribed an analgesic medicine for seven infants and seven infants received comfort interventions to manage the distress symptoms. Forty-one infants had a preexisting Do Not Resuscitate (DNR) order before they died. In 30 cases, both parents were with the infant and most often were holding their infants when death occurred. A quiet and isolated place was provided for these parents and their dying infants.

Conclusions: The research findings suggest that the application of the palliative care paradigm and more aggressive comfort care to manage symptoms might be beneficial to dying infants and their families in the NICU.

\section{NON-THERAPEUTIC INTERVENTIONAL RESEARCH IN PAEDIATRIC TRAUMA: WHAT DO PARENTS THINK?}

\author{
O. Davies, S. Thyaga Rajan
}

Paediatric Critical Care Unit, Barts and the London Children's Hospital, London, UK

Background: Emergency research in children in general is limited due to lack of robust informed consenting at such an emotional time for parents and families. These issues get even more magnified should such research involve non-therapeutic interventions particularly in the context of paediatric trauma.

Aim: To survey the voluntary opinion of parents about consenting for non-therapeutic interventional research in paediatric trauma.

Materials and Methods: We approached the parents of children seeking children's services at Barts and the London Children's Hospital, London to give voluntary opinion for retrospective consent for emergency blood tests studying early coagulation defects in children following trauma via a questionnaire.

Results: 66 parents agreed to give voluntary opinion and answered the questionnaire. 63 (95\%) parents were extremely supportive of the research project. Majority of the parents wondered why a research project to study these issues was not done yet. A few parents opined that they would support the research as long as essential emergency treatment is not compromised and consent is sought at the earliest suitable opportunity. Three $(5 \%)$ parents were unable to support the delayed consent issues since they felt it is too emotional a time to consider research.

Conclusions: Majority of the parents are willing for their children to take part in non-therapeutic interventional study despite the limitations of retrospective consenting in paediatric trauma. 